\title{
Nonhydrodynamic Collective Processes in Molten Salts: Theory and Ab Initio Simulations
}

\author{
TARAS BRYK ${ }^{1,2}$, IHOR MRYGLOD ${ }^{1,2}$ \\ ${ }^{1}$ Institute for Condensed Matter Physics of the National Academy of Sciences of Ukraine, \\ 1 Svientsitskii Street, UA-79011 Lviv, Ukraine \\ ${ }^{2}$ Institute of Applied Mathematics and Fundamental Sciences, Lviv National Polytechnic University, \\ UA-79013 Lviv, Ukraine
}

Received 31 October 2008; accepted 23 April 2009

Published online 17 August 2009 in Wiley InterScience (www.interscience.wiley.com).

DOI 10.1002/qua.22327

\begin{abstract}
We review recent progress in theory and ab initio simulations of collective dynamics in molten salts. Main focus is made on the dispersion of nonhydrodynamic optic-like excitations. A concept of "bare" propagating modes is used for the analysis of polarization effects on the dispersion of longitudinal optic modes. $\mathrm{Ab}$ initio molecular dynamics in combination with the generalized modes approach is used for consistent calculations of collective excitations spectra in molten $\mathrm{NaI}, \mathrm{LiF}$, and RbF. (c) 2009 Wiley Periodicals, Inc. Int J Quantum Chem 110: 38-45, 2010
\end{abstract}

Key words: collective excitations; ab initio molecular dynamics; molten salts; dispersion law

\section{Introduction}

$\mathbf{T}$ heoretical treatment of collective dynamics in liquids is a complicated problem because of the existence of different time scales of microscopic processes. Only on macroscopic spatial and temporal scales one can obtain correct analytical expressions

Correspondence to: T. Bryk; e-mail: bryk@icmp.lviv.ua

Contract grant sponsor: State Foundation for Fundamental Research of Ukraine.

Contract grant number: $\Phi 28.2 / 038$.

Contract grant sponsor: National Academy of Sciences of Ukraine. for time correlation functions $F_{i j}(k, t)$ with $i, j$ corresponding to conserved quantities for sufficiently small wavenumbers $k$ and large times. It is important that within such approach one can estimate the origin of the leading long-time processes contributing to the shape of $F_{i j}(k, t)$ and corresponding spectral functions, known as dynamical structure factors $S_{i j}(k, \omega)$ with $\omega$ being frequency, some of which can be measured in the X-ray or neutron scattering experiments [1]. For the case of binary liquids and molten salts, in particular, four hydrodynamic equations reflect in fact the local conservation laws and describe only the dynamical processes on large spatial and temporal scales comparing with average 
interatomic distance and characteristic molecular time, i.e., when the liquids are treated as continuum without any details of their atomic structure. Beyond the hydrodynamic region, where usually are located the wavenumbers available in scattering experiments and computer molecular dynamics (MD) experiments, the short-time processes with finite lifetime and a spatial scale of order of $10 \AA$ become very important and can significantly contribute to the shape of dynamical structure factors. Therefore, a lot of efforts have been made to derive analytical expressions for the hydrodynamic time correlation functions (in particular, for the densitydensity time correlation function $\left.F_{n n}(k, t)\right)$ that generalize the known hydrodynamic results and could be used beyond the hydrodynamic region. However, there is still a lack of clear understanding of the mechanisms of spectra formation in binary liquids. In general, there are just a few analytical studies in the literature, which would provide analytical expressions for dispersion laws of collective excitations in binary liquid systems. The most advanced theoretical results for the collective modes in binary liquids were obtained within the viscoelastic approximation of the memory function formalism $[2,3]$ and by the generalized collective modes (GCM) approach $[4,5]$. The viscoelastic approximation in general is valid for quite large wavenumbers and in the longwavelength limit is based on some assumptions for relevant correlation times.

In this work, we study collective dynamics in binary liquids using an approach of generalized hydrodynamics known as the method of GCM [4-6], in which the collective excitations in liquids are defined as the poles of relevant Green functions. The main feature of this approach is the existence of so-called kinetic collective excitations, which have finite lifetime and together with generalized hydrodynamic excitations contribute to the shape of the density-density time correlation functions $F_{n n}(k, t)$ beyond the hydrodynamic region. As the GCM approach takes into account the mode coupling effects in local approximation, i.e., interaction of hydrodynamic and kinetic excitations with the same wavenumber $k$, one can obtain very precious information about the dispersion law and the damping of collective excitations studied within an analytical treatment of different models in the GCM approach. For the case of binary liquids, the GCM approach permitted to obtain correct analytical expressions for the long-wavelength asymptotes of dispersion and damping of the transverse [7] and longitudinal [8] optic-like excitations.
The generalized hydrodynamic models within the GCM approach were successfully applied to analysis of time correlation functions derived in classical MD simulations of pure and binary liquids, and molten salts in particular $[9,10]$. To date, such an analysis is perhaps one of the most precise methods of estimation of leading wavenumber-dependent contributions to the time correlation functions of binary liquids, providing the precision of at least fourth frequency moments of partial dynamic structure factors. With the development of ab initio MD simulations, it became possible to estimate from the first principles the dispersion and damping of collective excitations in molten metals, alloys, and molten salts. However, so far only the simplest methodologies of spectra estimation such as analysis of peak positions of partial spectral functions or fit to the hydrodynamic expressions were used in combination with ab initio MD simulations. In this report, we aimed to show that it is possible to apply more precise methods of ab initio estimation of spectra of collective excitations in liquids such as the GCM approach. This is especially important for the case of molten salts, because, namely, ab initio simulations can correctly reproduce polarization effects without any constraints on effective charges and high-frequency dielectric permittivity.

The remaining article is organized as follows: in the next section we give some general remarks on the GCM approach and details of our MD simulations; in Section 3, an analytical solution of a simplified fourvariable dynamical model of binary liquids is given and discussed; results of ab initio molecular dynamics and GCM analysis of time correlation functions are given in Section 4; and in the last section, we summarize the results of this study.

\section{Method}

\subsection{GENERALIZED COLLECTIVE MODES APPROACH}

The GCM approach consists in solving of the generalized Langevin equation in matrix form in terms of dynamical collective eigenmodes of the liquid system. In fact, it is a parameter-free analysis of MD-derived time correlation functions by estimating dynamical collective eigenmodes in the liquid as the eigenvalues of generalized hydrodynamic matrix $\mathbf{T}(k)$, defined in Markovian approximation as follows [4, 11]:

$$
\mathbf{T}(k)=\mathbf{F}(k, t=0) \tilde{\mathbf{F}}^{-1}(k, z=0),
$$


where $\mathbf{F}(k, t)$ is the matrix of time correlation functions $F_{i j}(k, t)=\left\langle A_{i}(k, 0) A_{j}^{*}(k, t)\right\rangle$ constructed on a chosen basis set of $N_{v}$ dynamical variables $\mathbf{A}^{\left(N_{v}\right)}(k, t)$, and $\tilde{\mathbf{F}}(k, z)$ is its Laplace transform. The most general basis set of dynamical variables consists of the hydrodynamic variables and their time derivatives, which are supposed to describe correctly short-time processes. The time evolution of all dynamical variables can be obtained in MD simulations with the purpose of parameter-free estimation of elements of the matrix $\mathbf{F}(k, t)$. All the extended dynamical variables are usually generated as the $n$-th time derivative of partial current or energy density and can be obtained in analytical form and simply calculated from MD data.

The hydrodynamic variables of partial densities, total current density, and other relevant extended variables are sampled in MD to calculate matrix elements of the generalized hydrodynamic matrix $\mathbf{T}(k)$ (1). The eigenvalues of $\mathbf{T}(k)$ obtained at different wavenumbers formed the spectra of collective excitations.

Within the GCM method, there is no need to adopt some approximations for the higher order memory functions, because the dynamical correlations between different microscopic processes are incorporated in the theory via the generalized relaxation times associated with different kinds of hydrodynamic correlations in the system:

$$
\tau_{i j}(k)=\frac{1}{F_{i j}(k, 0)} \int_{0}^{\infty} F_{i j}(k, t) d t,
$$

where $F_{i j}(k, t)$ are the relevant hydrodynamic time correlation functions. The equivalence of the correlation times $\tau_{i j}(k)$ for MD-derived time correlation functions and their GCM replicas generates additional sum rules in our theoretical scheme [4]. Besides, the GCM replicas of corresponding time correlation functions can be represented in the separable form:

$$
F_{i j}^{\left(N_{v}\right)}(k, t)=\sum_{\alpha=1}^{N_{v}} G_{i j}^{\alpha}(k) e^{-z_{\alpha}(k) t},
$$

where each term corresponds to a separated contribution from the collective mode $z_{\alpha}(k)$.

\subsection{AB INITIO MOLECULAR DYNAMICS SIMULATIONS}

The ab initio simulations of $\mathrm{NaI}$ at $1080 \mathrm{~K}$ and numerical density $n=0.021 \AA^{-3}, \mathrm{LiF}$ at $1282 \mathrm{~K}$ and $n=0.0803 \AA^{-3}$, and $\mathrm{RbF}$ at $1115 \mathrm{~K}$ and $n=0.0331$

$\AA^{-3}$ were performed on Born-Oppenheimer surface (by the VASP package) having a collection of 150 particles for each molten salt. In all simulations a cubic box with the periodic boundary conditions and the NVT ensemble were used. The simulations for $\mathrm{NaI}, \mathrm{LiF}$, and $\mathrm{RbF}$ were performed with the generalized gradient approximation in Perdew-Wang-91 formulation [12], the electron-ion interactions were represented by the Kresse-Hafner ultrasoft pseudopotentials [13], and the highest default cut-off energy for them was used. Electronic density was constructed using a single $\Gamma$-point in Brillouin zone. The time step was $1.5 \mathrm{fs}$, and each configuration was saved for subsequent calculations of statistic averages. The number of configurations used for the estimation of static and time correlation functions ranged from 36,000 (for NaI) up to 51,700 (for $\mathrm{LiF})$, that is much larger numbers, than the usually reported in ab initio simulations of liquids. This resulted in very well converged tails of the time correlation functions, which is needed for the estimation of the relevant correlation times within the GCM approach. In Figure 1, we show the partial density autocorrelation functions for molten $\mathrm{LiF}$ at three different wavenumbers. One can see that even for small wavenumbers the partial density-density time correlation functions decay to zero within $\sim 8 \mathrm{ps}$, and the tails of the partial density-density time correlation are well converged.

For the estimation of polarization effects, additional classical molecular dynamics simulations were performed with the rigid-ion Tosi-Fumi model (similar as in Ref. [10]) at the same thermodynamic points.

\section{3. "Bare" Propagating Modes in Binary Liquid Systems}

In contrast to ideal crystals, where the phonons have formally infinite lifetime in harmonic approximation, the collective excitations in liquids always have some damping coefficients, which define their lifetime. However, in the case of liquid systems it is still very useful to perform initially an analysis of the dispersion law on the basis of simple analytical expressions for a reference dispersion law of collective excitations, obtained exactly within some simplified dynamical models. This dispersion law corresponds to the so-called "bare" collective modes. On the next step one can estimate the changes in the 


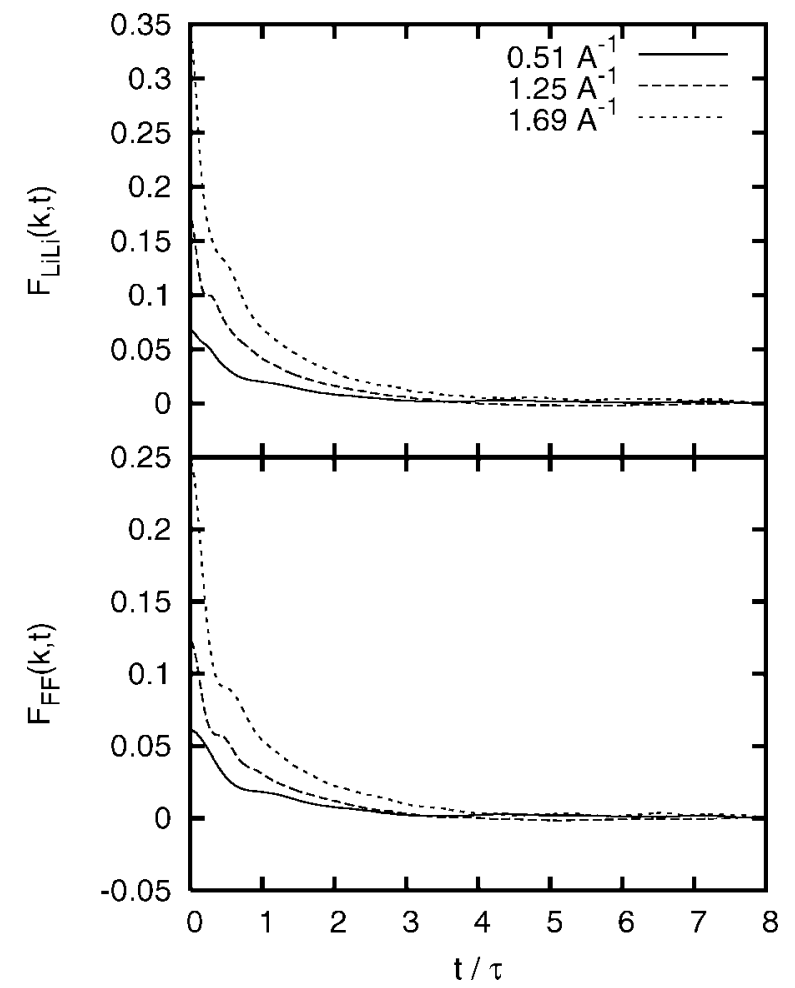

FIGURE 1. Partial density autocorrelation functions for molten LiF at $1282 \mathrm{~K}$ for three wavenumbers. The well-converged tails of time correlation functions enabled to obtain the wavenumber-dependent correlation times (2), which are the input quantities in the parameter-free GCM approach. The time unit $\tau$ is equal to 0.217 ps.

dispersion of collective modes caused by the coupling effects with some relaxation processes. Such a scheme was successfully applied in a study of structural relaxation in pure liquids [14], where the coupling of the nonhydrodynamic structural relaxation process with the "bare" hydrodynamic sound mode led to a "positive dispersion" of the sound branch. For the case of molten salts the "bare" collective modes are of special interest, because namely the "bare" frequencies of longitudinal (LO) and transverse (TO) optic modes should enter a sum rule for ionic melts [15], which corresponds to the LyddaneSachs-Teller relation known for ionic crystals [16]. This sum rule reads

$$
\left[\omega_{\mathrm{LO}}^{0}(k=0)\right]^{2}-\left[\omega_{\mathrm{TO}}^{0}(k=0)\right]^{2}=\omega_{\mathrm{p}}^{2}=\sum_{\nu} \frac{4 \pi n_{\nu} z_{v}^{2} e^{2}}{m_{v}},
$$

where $\omega_{\mathrm{p}}$ is the plasma frequency, $n_{v}, m_{v}$, and $z_{v}$ are the number density, atomic mass, and ionic charge of the corresponding species.
A simplified model of collective dynamics in binary liquids was suggested in Ref. [17]. This dynamical model can be called as an "elastic" one because it does not take into account directly the slow thermal and mutual diffusion processes in liquid; however, some microscopic quantities connecting with the forces acting on particles (and therefore reflecting elastic properties) are present explicitly. In this case, the basis set of dynamical variables for the longitudinal dynamics includes four variables

$$
\mathbf{A}^{(4)}(k, t)=\left\{J_{\alpha}^{L}(k, t), J_{\beta}^{L}(k, t), \dot{J}_{\alpha}^{L}(k, t), \dot{J}_{\beta}^{L}(k, t)\right\},
$$

where the pair of indices $\{\alpha, \beta\}$ corresponds to two orthogonal currents, so that $\left\langle\mathbf{J}_{\alpha} \mathbf{J}_{\beta}\right\rangle=0$, and the overdots mean the first time derivatives of the relevant dynamical variables. It is convenient to consider as such pairs of orthogonal currents the partial mass currents

$$
\mathbf{J}_{\alpha}(k, t)=\frac{1}{\sqrt{N}} \sum_{i=1}^{N_{\alpha}} m_{\alpha} \mathbf{v}_{i, \alpha}(t) e^{i \mathbf{k r}_{i, \alpha}(t)}, \quad \alpha=A, B,
$$

or the linear combinations of partial currents that describe the total mass $\mathbf{J}_{t}(k, t)$ and massconcentration $\mathbf{J}_{x}(k, t)$ currents [17]. In (6) the $\mathbf{r}_{i, \alpha}$ and $\mathbf{v}_{i, \alpha}$ are the position and velocity of the $i$-th particle in the $\alpha$-th species. The generalized kinetic matrix $\mathbf{T}^{(4)}(k)$ is easily obtained for the basis set $\mathbf{A}^{(4)}(k, t)$, introduced above. One has

$$
\mathbf{T}^{(4)}(k)=\left(\begin{array}{cccc}
0 & 0 & -1 & 0 \\
0 & 0 & 0 & -1 \\
T_{31}(k) & T_{32}(k) & 0 & 0 \\
T_{41}(k) & T_{42}(k) & 0 & 0
\end{array}\right)
$$

with the matrix elements

$$
\begin{aligned}
T_{31}(k)=\frac{f_{j_{\alpha} j_{\alpha}}}{f_{J_{\alpha} J_{\alpha}}}, \quad T_{32}(k)=\frac{f_{j_{\alpha} j_{\beta}}}{f_{J_{\beta} J_{\beta}}}, \\
T_{41}(k)=\frac{f_{j_{\beta} j_{\alpha}}}{f_{J_{\alpha} J_{\alpha}}}, \quad T_{42}(k)=\frac{f_{j_{\beta} j_{\beta}}}{f_{J_{\beta} J_{\beta}}}
\end{aligned}
$$

where

$$
f_{A B}(k)=\langle A(k) B(-k)\rangle
$$

are the static correlators between corresponding dynamical variables. For the generalized kinetic matrix (7), it is straightforward to obtain the 


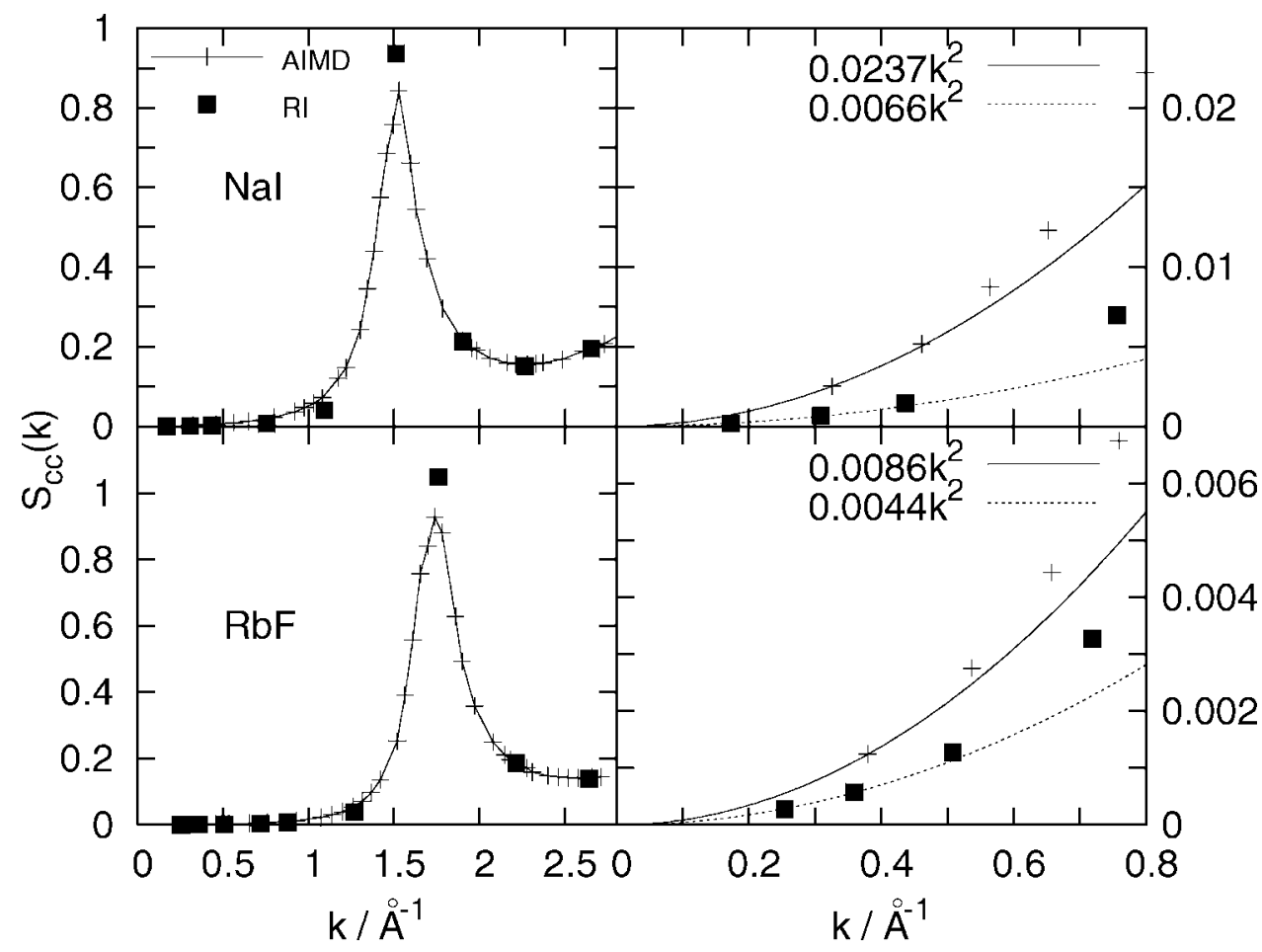

FIGURE 2. Concentration-concentration static structure factors for molten $\mathrm{Nal}$ and $\mathrm{RbF}$, obtained from the ab initio (AIMD) and classical MD with rigid-ion model. The long-wavelength asymptotes are shown in the right frames.

eigenvalues that correspond to two pairs of propagating collective modes:

$$
\begin{aligned}
& z_{1}^{0}= \pm i\left[\frac{1}{2}\left(T_{31}+T_{42}\right)-\frac{1}{2} \sqrt{\left(T_{31}-T_{42}\right)^{2}+4 T_{32} T_{41}}\right]^{\frac{1}{2}} \\
& \equiv \pm i \omega_{1}^{0}(k) \\
& z_{2}^{0}= \pm i\left[\frac{1}{2}\left(T_{31}+T_{42}\right)+\frac{1}{2} \sqrt{\left(T_{31}-T_{42}\right)^{2}+4 T_{32} T_{41}}\right]^{\frac{1}{2}} \\
& \equiv \pm i \omega_{2}^{0}(k) \text {. }
\end{aligned}
$$

Note that these complex-conjugated eigenvalues are purely imaginary, i.e., they describe the propagating excitations without damping, because all the dissipation mechanisms are neglected in the actual elastic approximation. Nonzero damping coefficients (real parts of eigenvalues in the GCM approach) appear when the coupling of these "bare" propagating modes with relaxation processes in liquids, connected with diffusion, ionic conductivity, structural relaxation, etc. is taken into account. The expressions (8) describe the dispersion of two branches of "bare" collective excitations and can be used for both cases of orthogonal currents considered. It is important that in the whole range of wavenumbers such a dynamical model takes into account in appropriate way the effects of cross-correlations between the two propagating processes. We have to mention here that the analytical expressions (8) for "bare" collective modes, obtained within the GCM approach, yield identical results with the intuitive approach, based on the diagonalization of a matrix of second frequency moments of the partial current spectral functions, applied in [18] for the estimation of dispersion of collective excitations in a metallic glass.

\section{Optic-Like Collective Excitations in Molten Salts}

To estimate the effect of electron shell deformability, correctly reflected in the ab initio molecular dynamics simulations, on the optic-like collective modes in molten salts, we compared the static structure factors with those, obtained in classical MD simulations for rigid-ion model. We are mainly interested in the long-wavelength 
asymptotes of the concentration-concentration structure factors $S_{\mathrm{cc}}(k)=\left\langle n_{\mathrm{c}}(k) n_{\mathrm{c}}(-k)\right\rangle$ with

$$
n_{\mathrm{c}}(k, t)=\frac{1}{2}\left[n_{+}(k, t)-n_{-}(k, t)\right],
$$

which should tend in the long-wavelength limit as [19]

$$
\left.S_{\mathrm{cc}}(k)\right|_{k \rightarrow 0} \sim \frac{\varepsilon k_{\mathrm{B}} T}{4 \pi n e^{2}} k^{2},
$$

where $\varepsilon$ describes the effects of bare charge screening due to the electron shell deformability, $n$ and $e$ correspond to number density of the system and the elementary charge, respectively. It is clear that for the rigid-ion model one has $\varepsilon=1$, while in $\mathrm{ab}$ initio molecular dynamics the effects of electron cloud deformability are taken into account explicitly. Hence, by comparing both long-wavelength asymptotes one can estimate the value of $\varepsilon$ simply by taking the ratio of the coefficients of $k^{2}$ terms, because the density and temperature in the $\mathrm{ab}$ initio and rigid-ion simulations were identical for all the molten salts considered in this study. In Figure 2 we show the concentration-concentration structure factors for $\mathrm{NaI}$ and $\mathrm{RbF}$, obtained from the classical and ab initio simulations. Note that the short-wavelength asymptote for the $S_{\mathrm{cc}}(k)$ is 0.25 . On the right frames of Figure 2 one can see the corresponding long-wavelength asymptotes of calculated structure factors $S_{\mathrm{cc}}(k)$, which permit estimation of the high-frequency dielectric permittivity $\varepsilon$. The obtained values of $\varepsilon$ and corresponding experimental data for the high-frequency dielectric permittivity of ionic crystals are shown in Table 1 . The estimated values of $\varepsilon$ for $\mathrm{NaCl}$ [17], $\mathrm{LiF}$, and $\mathrm{RbF}$ are in very good agreement with the high-frequency dielectric permittivity of corresponding ionic crystals, while for the case of molten $\mathrm{NaI}$ we obtained a discrepancy

\section{TABLE I}

\section{Experimental data for high-frequency dielectric permittivity of ionic crystals [16] and values $\varepsilon$ obtained in ab initio molecular dynamics studies for four molten salts.}

\begin{tabular}{lcc}
\hline System & Exp. & AIMD \\
\hline $\mathrm{NaCl}[17]$ & 2.20 & 2.22 \\
$\mathrm{Nal}$ & 3.01 & 3.59 \\
$\mathrm{LiF}$ & 1.93 & 1.95 \\
$\mathrm{RbF}$ & 1.93 & 1.95 \\
\hline
\end{tabular}

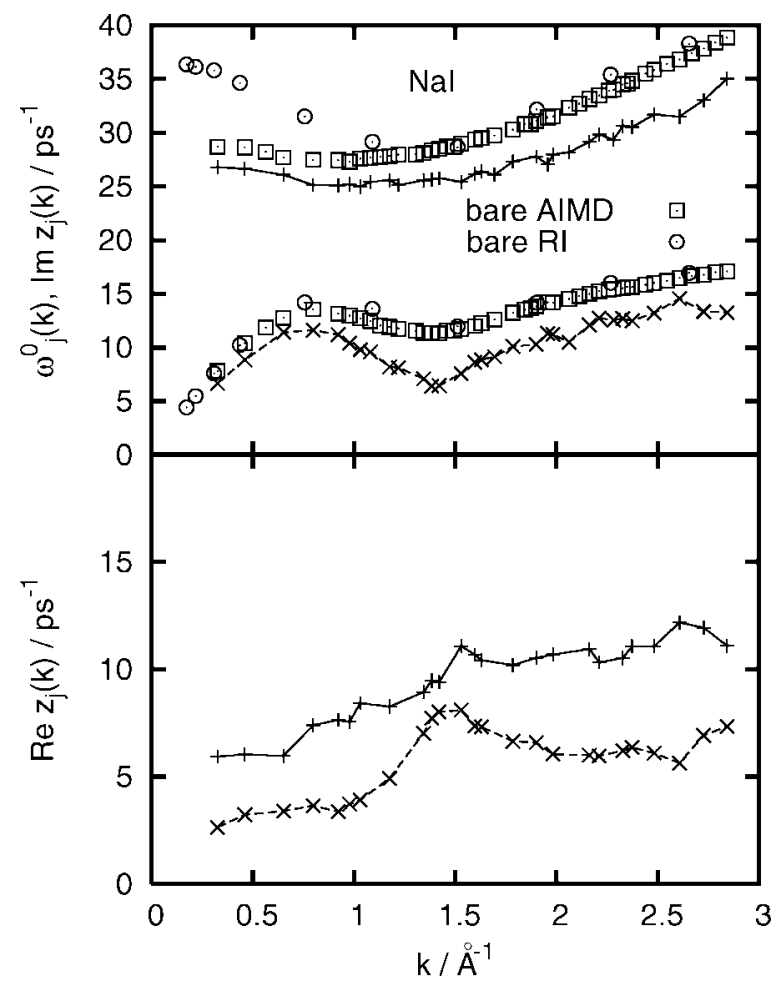

FIGURE 3. Dispersion (top frame) and damping (bottom frame) of collective excitations (line-connected symbols "plus" and "cross") in molten Nal at $1080 \mathrm{~K}$, obtained for the set of dynamical variables (10) on the basis of ab initio simulations. The high- and low-frequency branches correspond to the optic and acoustic modes in long-wavelength region. Dispersion of "bare" collective modes qualitatively reproduces the $k$-dependence of the branches of collective excitations. Because of the polarization effects, the "bare" frequency of optic-like modes is softened in comparison with the rigid-ion case.

of $\sim 15 \%$ in comparison with the case of crystal, that perhaps is the consequence of large polarizability of mainly $\mathrm{I}^{-}$ions, which contain eight electrons in the outer shell and are much larger than the other studied here anions. It is also possible that in the melt the polarizability of ions can be larger than in the crystal; however, this needs to be verified by independent calculations. Here, we would also like to remind that the molten salts are ionic conductors, but electronic insulators, i.e., the static dielectric permittivity in molten salts is formally infinite.

The polarization effects essentially affect the dispersion of optic-like branch in the long-wavelength region. This can be clearly seen in Figure 3 by comparing the "bare" frequencies of collective modes for molten $\mathrm{NaI}$, obtained from classical rigid-ion 


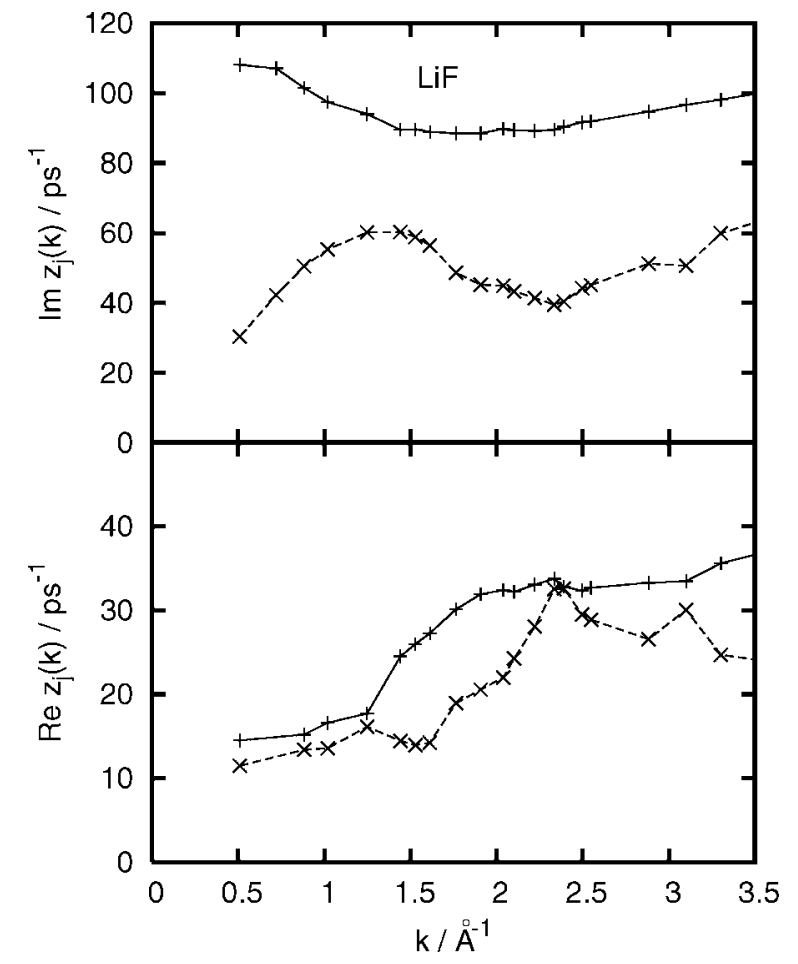

FIGURE 4. Dispersion (top frame) and damping (bottom frame) of collective excitations in molten LiF at $1282 \mathrm{~K}$, obtained for the set of dynamical variables (10) on the basis of ab initio simulations.

simulations (RI) and ab initio molecular dynamics (AIMD). The perfect match of low-frequency "bare" modes from both simulations makes evidence that there were no essential size effects from different MD boxes used in classical and ab initio simulations. It is well seen in Figure 3 that only the high-frequency branch softens in the region $k<1 \AA^{-1}$, which can be ascribed to the screening of the inner electric field of moving in the opposite direction ions $\mathrm{Na}$ and I by polarization of the electronic density. The "bare" modes qualitatively correctly reproduce the dispersion of both high- and low-frequency branches of collective excitations $z_{j}(k)=\sigma_{j}(k)+i \omega_{j}(k)$, which were obtained on the six-variable set of dynamical variables

$$
\begin{aligned}
& \mathbf{A}^{(6)}(k, t) \\
& \quad=\left\{n_{+}(k, t), n_{-}(k, t), J_{+}^{L}(k, t), J_{-}^{L}(k, t), \dot{J}_{+}^{L}(k, t), \dot{J}_{-}^{L}(k, t)\right\},
\end{aligned}
$$

that corresponds to the viscoelastic treatment of collective dynamics. Completely in agreement with the concept of "bare" collective modes, we found the reduction of the "bare" frequencies due to coupling with the slow relaxation processes, represented by the fluctuations of partial number densities in the basis set $\mathbf{A}^{(6)}(k, t)$. The collective excitations have obtained nonzero damping $\sigma_{j}(k)$, shown in the bottom frame of Figure 3.

The spectra of collective excitations in molten $\mathrm{LiF}$ at $1282 \mathrm{~K}$ and $\mathrm{RbF}$ at $1115 \mathrm{~K}$ are shown in Figures 4 and 5 . The propagating modes were obtained within the same six-variable viscoelastic model $\mathbf{A}^{(6)}(k, t)$ on the basis of our ab initio simulations. Essentially higher frequencies of the optic-like mode in $\mathrm{LiF}$ in comparison with molten $\mathrm{NaI}$ are due to much smaller atomic mass of salt components. The rigidion simulations of molten $\mathrm{LiF}$ were reported in [9]. Here, we can mention that the frequency of longwavelength optic modes for $\mathrm{LiF}$ has softened from $\sim 135 \mathrm{ps}^{-1}$ for the rigid ions down to $\sim 110 \mathrm{ps}^{-1}$ due to the electron polarization effects.

The damping of collective excitations, which in the GCM approach corresponds to the real part of complex-conjugated eigenvalues, is shown in the bottom frames of Figures 4 and 5. Similar, as in the case of $\mathrm{NaI}$ the damping of the high-frequency

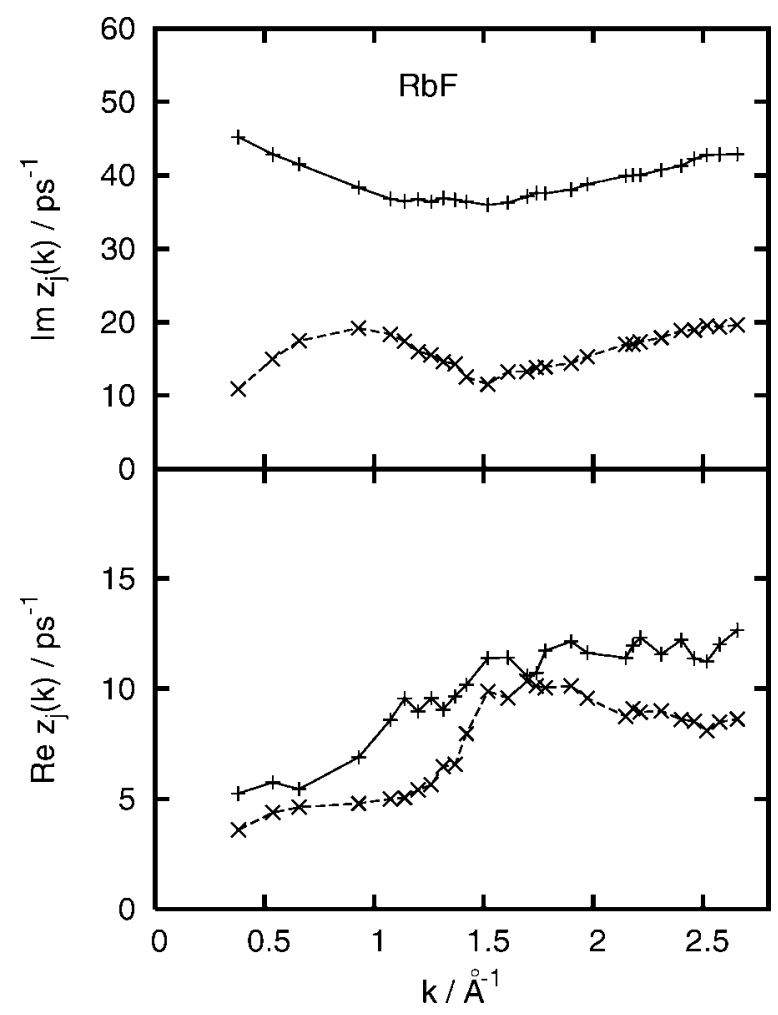

FIGURE 5. Dispersion (top frame) and damping (bottom frame) of collective excitations in molten $\mathrm{RbF}$ at $1115 \mathrm{~K}$, obtained for the set of dynamical variables (10) on the basis of ab initio simulations. 
branches is higher, than the damping of acoustic excitations. The low-frequency branch has the maximum of damping right at the locations of the roton-like minima in dispersion curves. In the long-wavelength region, the damping of nonhydrodynamic opticlike modes tend to nonzero values, which means finite lifetimes on macroscopic spatial scales, i.e., the nonhydrodynamic modes do not survive on macroscopic distances in contrast to acoustic excitations.

\section{Summary}

We reported a combination of analytical and $\mathrm{ab}$ initio study of dispersion and damping of collective modes in molten salts. The analysis of dispersion of "bare" collective modes and comparison of their frequencies with the rigid-ion calculations permits to observe clearly the effects of ion polarization on the dispersion curves of collective modes. The longitudinal optic-like branch softens due to electronic density polarization, which can be quite correctly reproduced by the ab initio molecular dynamics, as it was shown on the example of estimation of high-frequency dielectric permittivity of molten salts. The theoretical approach used in this study permits to estimate the spectra of generalized hydrodynamic as well as nonhydrodynamic collective excitations. These modes contribute with different weights, which can be also estimated within the GCM approach, to the time correlation functions obtained in ab initio molecular dynamics, that is very important for the explanation of experimental IXS intensities measured recently for molten $\mathrm{NaCl}$ [20], $\mathrm{NaI}$ [21], LiF (Pontecorvo, unpublished), and RbF (Masciovecchio, private communication).

\section{ACKNOWLEDGMENTS}

Allocation time at the Center for Supercomputer Calculations at Kiev National Technical University is acknowledged. The calculations have been performed by using the ab initio total energy and molecular dynamics program VASP (Vienna ab initio simulation program) developed at the Institute für Materialphysik of the Universität Wien [22-24].

\section{References}

1. Scopigno, T.; Ruocco, G.; Sette, F. Rev Mod Phys 2005, 77, 881.

2. Chushak, Y.; Bryk, T.; Baumketner, A.; Kahl, G.; Hafner, J. Phys Chem Liq 1996, 32, 87.

3. Anento, N.; Gonzalez, L. E.; Gonzalez, D. J.; Chushak, Y.; Baumketner, A. Phys Rev E 2004, 70, 041201.

4. Mryglod, I. M.; Omelyan, I. P.; Tokarchuk, M. V. Mol Phys 1995, 84, 235.

5. Bryk, T.; Mryglod, I.; Kahl, G. Phys Rev E 1997, 56, 2903.

6. de Schepper, I. M.; Cohen, E. G. D.; Bruin, C.; van Rijs, J. C.; Montfrooij, W.; de Graaf, L. A. Phys Rev A 1988, 38, 271.

7. Bryk, T.; Mryglod, I. J Phys Condens Matter 2000, 12, 6063.

8. Bryk, T.; Mryglod, I. J Phys Condens Matter 2002, 14, L445.

9. Bryk, T.; Mryglod, I. J Phys Condens Matter 2004, 16, L463.

10. Bryk, T.; Mryglod, I. Phys Rev B 2005, 71, 132202.

11. Mryglod, I. M. Condens Matter Phys 1998, 1, 753.

12. Perdew, J. P.; Wang, Y. Phys Rev B 1992, 45, 13244.

13. Kresse, G.; Hafner, J. J Phys Condens Matter 1994, 6, 8245.

14. Bryk, T.; Mryglod, I. Condens Matter Phys 2008, 11, 138.

15. O'Sullivan, K. F.; Madden, P. A. J Phys Condens Matter 1991, 3, 8751 .

16. March, N. H.; Tosi, M. P. Coulomb Liquids; Academic Press: London, 1984.

17. Bryk, T.; Mryglod, I. Chem Phys Lett 2008, 466, 56.

18. Hafner, J. J Phys C 1983, 16, 5773.

19. Rovere, M.; Tosi, M. P. Rep Prog Phys 1986, 49, 1001.

20. Demmel, F.; Hosokawa, S.; Lorenzen, M.; Pilgrim, W.-C. Phys Rev B 2004, 69, 012203.

21. Demmel, F.; Hosokawa, S.; Pilgrim, W.-C.; Tsutsui, S. Nucl Instrunt Methods B 2005, 238, 98.

22. (a) Kresse, G.; Hafner, J. Phys Rev B 1993, 47, 558; (b) Kresse, G.; Hafner, J. Phys Rev B 1994, 49, 14251.

23. Kresse, G.; Furthmüller, J. Comput Mater Sci 1996, 6, 15.

24. Kresse, G.; Furthmüller, J. Phys Rev B 1996, 54, 11169. 